\title{
Model Predictive Controller based Unified Power Quality Conditioner for Voltage Regulation Studies in 33- Bus Closed Loop Distribution System
}

\author{
SK. Abdul Pasha ${ }^{1}$, N. Prema Kumar ${ }^{2}$ \\ ${ }^{1}$ Research-Scholar and Assistant Professor, Départment of EEE, Anurag Engineering Collège (A), Kodad, India \\ ${ }^{2}$ Professor-Dept. of Electrical Engineering, Andhra University College of Engineering (A), Vskp, India
}

\begin{abstract}
Recent developments in FACTS have produced U.P.Q.C to mitigate sag and attenuate THD. U.P.Q.C has been urbanized as a FACTS controller between feeding end \& far end of distribution system .The U-P-Q-C is capable of improving the voltage profile \& reducing THD of distribution system by regulating the voltage using PR (Proportional-Resonant-Controller) and MPC (Model-Predictive) controller. This work proposes U-P-Q-C for Thirty Three Bus Systems. The objective of this work is to enhancevoltage-profile of T-T-B-S. The T-T-B-S in open loop \& closed loop-TTBS- U-P-Q-C using PR and MPCcontrollers are-modeled,pretend $\&$ their consequences are represented. Responses are estimated as a time of settle and error in steady state. The outcomes indicate that MP Controlled T-T-B-S system has better response than PR controlled T-T-B-S system.
\end{abstract}

\section{Introduction}

Ideal mode of power networks in altering receiving end responses be a tricky issue. For regular network modules most of the substiutional elements introduced for enlarging functionality of respective devices. Just like bank of capacitors, loading taps, potential controllers etc.Evaluation of ideal locations of respective elements for such apparatus for regulating responses requires formulization of in service optimal approach. Effect over power-energy to be the most affordable parameter in practice to identify locations of substitution elements [14].General case of withholding devices be insertion of capacitors [1-2],re configural of networks[3],allocating DG's[4] etc.Even though entair methods will make PEreduction, over it will be complications of adopting such innovations. Fixing capacitors be a simplest mode to dominant loss reduction of energy. When secondary end is alternative in nature, finest regulation is difficult.

Likewise set of capacitors are modulated with simplest procedures [2].Further variations in positional aspects from single point to some other develops interruptions. Identifying a system response will goes with suitable deployment of E-loss. PQ-issue will be treated as a un-standard-voltage, current/frequency which exhibits a mal-operation for recieving end devices. User level distribution-models, smoothen industrial-loads and crucial commercial-operations all effects from various levels of outages and service ditortions which makes suitable damage for incidents suited in running-down-time, mis match-generation, similar task-models and other parameters [5]. The paper over-whelms about power quality conditioner(PQC), it suits to execution towards series-shunt APFs. Objective of the UPQC is to substitute element as for potential flickers/im-balances, reactive-powers, opposite-sequence currents and harmonies. Other side, it has the ability to improve quality of power at installation sectors on distribution-networks or industrial-power-systems [6].

It is pretended to represent a wide variety of suitable conditioner modules to 1-ph (2W) and 3-ph (3W and $4 \mathrm{~W})$ systems, various substitutional-models, latest innovations in the fields. It is mentioned that somanyresearchers will be considered various titles to conditioner suited to similar-function, tasks, applications or topologies with selection [7-11].S-PV arrays are integrates to DC-links for PQC-S-PV via simple-boostconverter. It is regulated by MPPT-algorithm for operating PV-array at top stream locations.seriescompensators-control will based on liberation of points of Common-Coupling-Voltage-Template for utilising 2 cascaded-SOGI-band-pass-filter.

Parllel-compensating-controller will depends on extractive fundamentals load- current. If load-current is highly un-linear, a mix of CSOGI\&DSC considered for extracting fundamentals in service element of load by that ref-signal for parllel-compensators are developed. Design and performance analysis of 3phase solar-PVintegrated-UPQC[12-13].1-phase-non-transformerconditioner-(NT-UPQC) will be represented. Non other than not any isolated-transformer, recommended blocks considers 4-switching-elements only, framing 2-half-

* Corresponding author: abdulpsh@gmail.com 
bridge VSI's, 1-connected in same line to load and other tied in-series via ac-mains.2-inverters takes similar dclinks.Shunt-inverters controls from a hysteresis-currentcontroller, is considered to alter the current drawn from ac-mains \& regulates the dc-link-voltage.

Modeling, insertion of 'Open-UPQC' integrated PVG-system for improving efficiency of energy \& PQof Radial-Distribution-Networks.Similar way UPQCOWOB, energy extracted from PVA will directly given to the model. Recommended models incorporates in forward\&backward-sweeps of load flows to evaluate operating-parameters like node-voltage [14-18].

The overhead literature does not pact with U-P-Q$\mathrm{C}$ for T-T-B-S. This investigation suggests a U-P-Q-C for T-T-B-S. This work proposes MPC for the control of T-T-B-S. The comparison of simulation - results with PR and MPC are presented. The overhead literature does not evaluate the-response of PRC and Model Predictive controlled TTB- systems. "This effort-covenants with evaluation of time-responses of PRC and Model Predictive controlled -T-T-B-Systems".

\section{System Configuration}

\subsection{Block Diagram}

The block diagram of U-P-Q-C system is outlined in Fig1.It is basically modeled with 2 major systems i.e. series and shunt inverters followed by a dc-linkcapacitor respectively. Initially the system is simulated in MATLAB/SIMULINK environment with 33buses.The effectiveness of proposed SM controller approach is illustrated using IEEE 33-bus system.

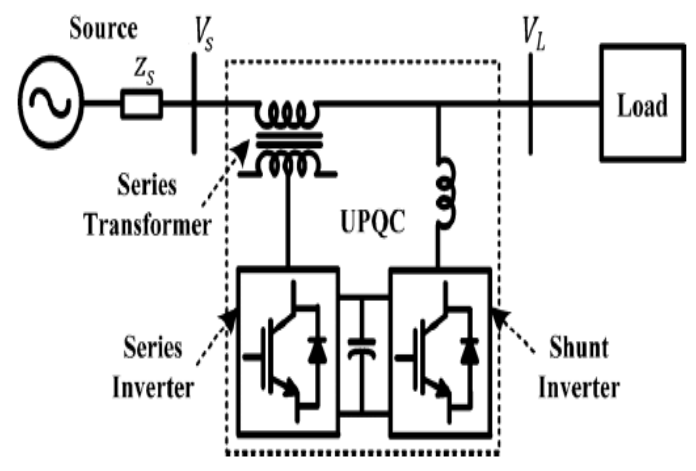

Fig. 1. Block-diagram-of a U-P-Q-C system

Block-diagram for closed loop PR controlled U-P-Q-C 33-bus-method is outlined in-Fig 2.It combines both SE$\mathrm{SH}$ technologies in it follwed by a suitable srorage system for immediate response for compensating voltages in it.

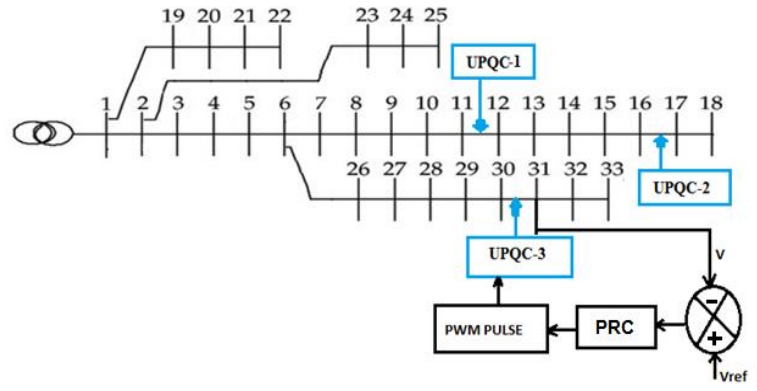

Fig. 2. Single line diagram of a closed loop PR Controlled UP-Q-C -T-T-B-S

Block-diagram of closed loop Model Predivtive controlled U-P-Q-C 33-bus-method is outlined in-Fig 3.

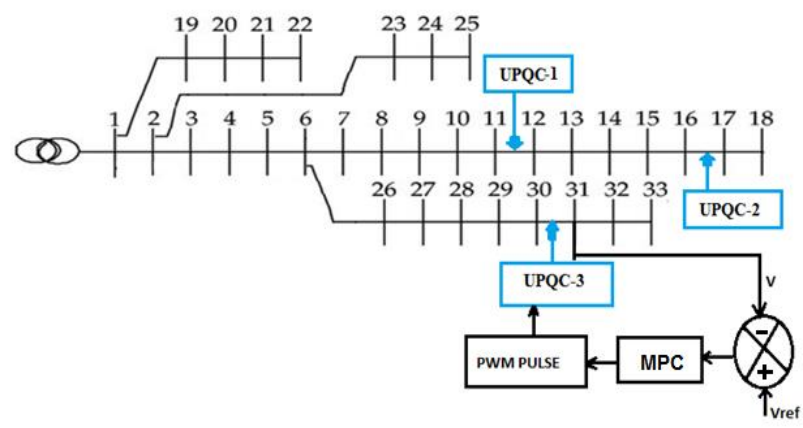

Fig. 3. Single line diagram of a closed loop MP Controlled U-PQ-C 33-bus system

\subsection{Design of U.P.Q.C System}

Acquiring the values of $\mathrm{V}_{1}, \mathrm{I}_{1}$ \& recurrence-of-MOSFEToffers the-scheme. Centered on-requisite-capacitivevoltages, 'duty-ratio' will assessed exploiting to eq ${ }^{\mathrm{n}}$

$$
\mathrm{V}_{0}=\frac{\mathrm{V}_{1}}{(1-a)}
$$

Proficiency-of-converters for estimating-yield-current as

$$
\eta=\frac{V_{a} I_{a}}{V_{1} I_{a}}
$$

Assessments of LC are estimated with considering $\Delta \mathrm{I}$ $\Delta \mathrm{V}$ as

$$
\begin{gathered}
\Delta \mathrm{V}=\frac{\mathrm{V}_{1} \mathrm{D}}{\mathrm{fL}} \\
\Delta \mathrm{I}=\frac{\mathrm{I}_{\mathrm{g}} \mathrm{D}}{\mathrm{fC}}
\end{gathered}
$$

Potential will permeated with indistinguishable of' I-Z'. Value of $\mathrm{C}_{5}$ be adopted and $\mathrm{L}_{5}$ is estimated with formulation

$$
f_{5}=\frac{1}{2 \pi\left(L_{5} C_{5}\right)^{\frac{1}{2}}}
$$

PW-value for DVR-AF inverter-switches as $\frac{T_{D}}{2}, \frac{T_{5}}{2}$

\subsection{Proportional Resonant Controller}

A tremendous response with I-controllers reflects as superlative response with minimum interruptions for avoiding harmonic contents. A proportional resonant control strategy is used as compensator to track a 
sinusoidal current reference frame. The basic control loop diagram [4] with PR control is as shown in figure 4.

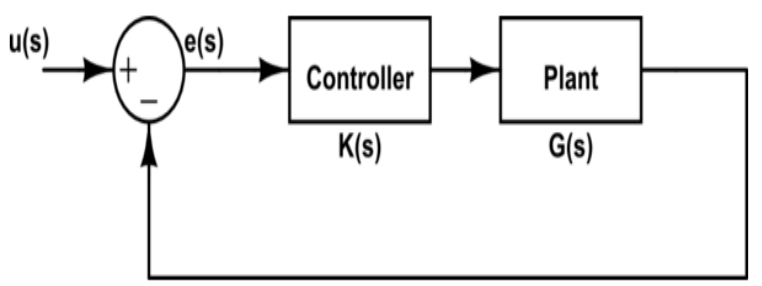

Fig. 4. Control loop diagram with PR controller

Transfer function of the ideal PR controller is as below:

Where,

$$
G_{P R}(s)=K_{p}+K_{R} \frac{s}{s^{2}+w_{o}^{2}}
$$

$K_{p}$ - proportional gain of the controller

$K_{R}-$ Resonant gain of the controller

$w_{o}-$ Resonant frequency of the controller in general

which is frequency of the grid.

An approximate ideal -PRC given by

Where

$$
G_{P R}(s)=K_{p}+K_{R} \frac{2 \omega_{c} s}{s^{2}+2 \omega_{c} s+w_{0}^{2}}
$$

$\omega_{c}-$ B.W of $w_{o}$

Resonant-peak holds finite-gain of $40 \mathrm{~dB}$ it will suitably huge to eliminate potential track-error. Some other larger band-width will be noticed surrounds the resonantfrequency, it reduces smoothness of controller to little grid level frequency-variations. Other side harmonyfrequencys, resultant of un-ideal PRC will matches with Genuine-PR controller.

\subsection{Model Predictive Controller (MPC)}

It uses a model for system to come across predictive surrounds system future-behaviour. It resolves in service optimized algorithms for evaluating optimal-control actions which derives predictive response to referal. The future conduct of the framework is founded with an improvement cost work dependent on subsiding skyline guideline utilizing current framework esteems. The main component of the determined info vector which is gotten each time step is applied to the framework as a control contribution inside a predefined streamlining window. The fundamental guideline of MPC is outlined in below figure 5 .

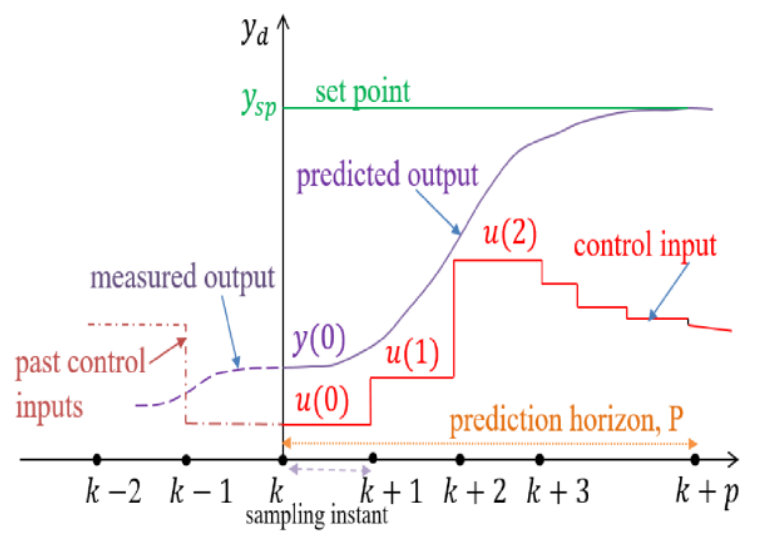

Fig. 5. Principle of-Model-Predictive-Control

Predective-Controller will be fabricated by optimisationcontrol blocks.

Let $\mathrm{s}_{0}=\mathrm{rh}$ and $\mathrm{t}=(\mathrm{r}+1) \mathrm{h}$ and let us use shorthand notation,

$$
\mathrm{m}(\mathrm{rh})=\mathrm{x}(\mathrm{r})
$$

The discrete output equation has the form for mpc will follows as,

$$
\mathrm{y}(\mathrm{r})=\mathrm{Cx}(\mathrm{r})
$$

\section{Simulation Results}

\subsection{Closed-loop-UPQC TTBC with PRC}

Fig.below delineates the Circuit for closed loopU.P.Q.C-based T-T-B-S with PRcontroller.Load-Voltage be sensed\&it will evaluated by-mentioned potential value to obtain Voltage-Errors'. The-voltage-inaccuracy -deviated for PRC. Yield of-PRC utilized for updating PW-Value. Fig. 7 outlines Voltage at bus-31 of the closed loop-UPQC-based T-T-B-S with PRcontroller.

The value of voltage at bus- 31 is $0.82 * 10^{4} \mathrm{~V}$. Fig .8 outlines the Current at bus-31 of the closed loop-UPQCbased T-T-B-S with PRcontroller. The value of Current at bus-31 is 90 A. Fig.9 outlines the RMS voltage at bus31 . The value of RMS voltage is $6300 \mathrm{~V}$ and it is stable. -Fig. 10 outlines the Real power at bus- 31 of the closed loop-UPQC-based T-T-B-S with PRcontroller. Real power-value- is $4.23 * 10^{5} \mathrm{MW}$ \&it is stable. Fig. 11 outlines the Reactive power at bus-31 of the closed loopUPQC-based T-T-B-S with PRcontroller. The value of Reactive power is $4.34 * 10^{4} \mathrm{MVAR}$ and it is stable. 


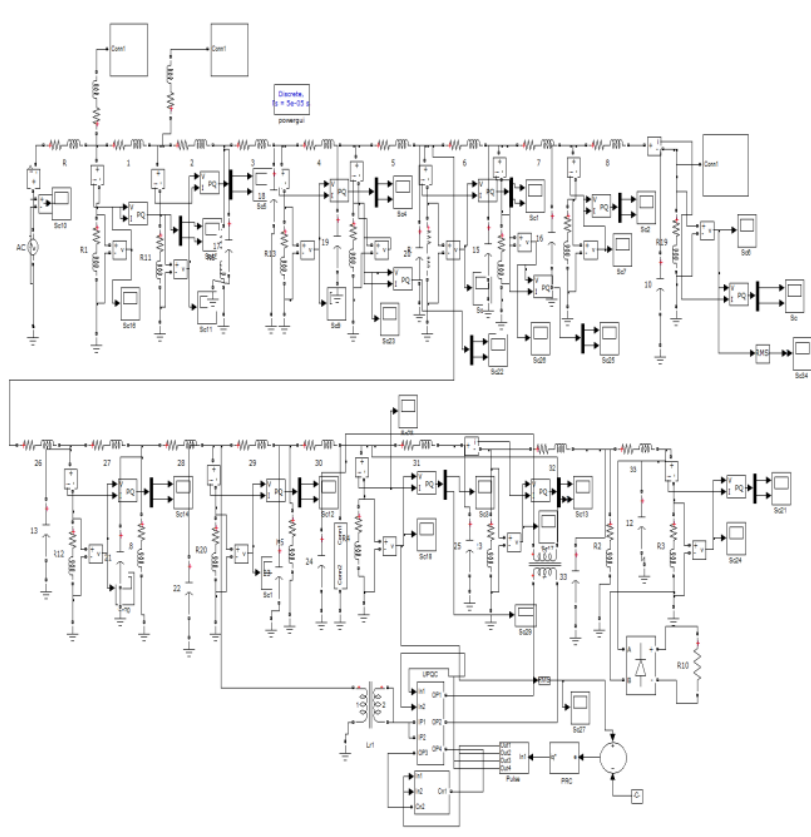

Fig. 6. Circuit diagram for CL-UPQC based-33bus with PR controller

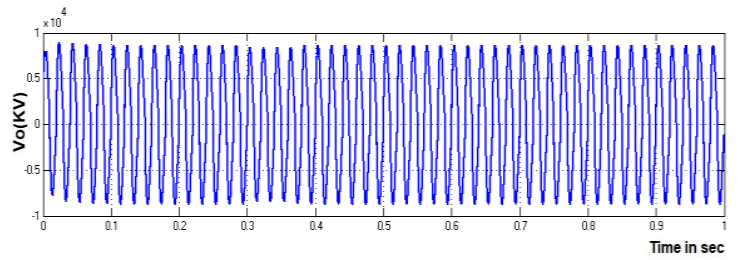

Fig. 7.Voltage at bus-31 for-CL -UPQC based-33-BUS with PR controller

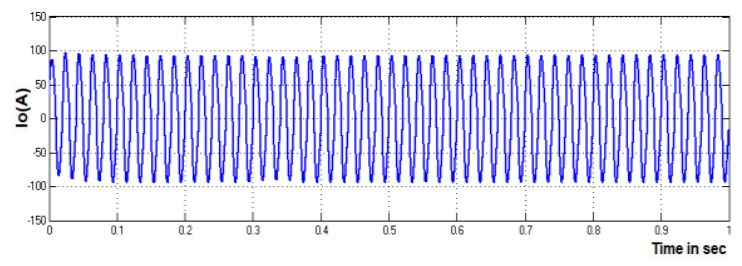

Fig. 8. Current at bus-31 toC-UPQC based PR controller

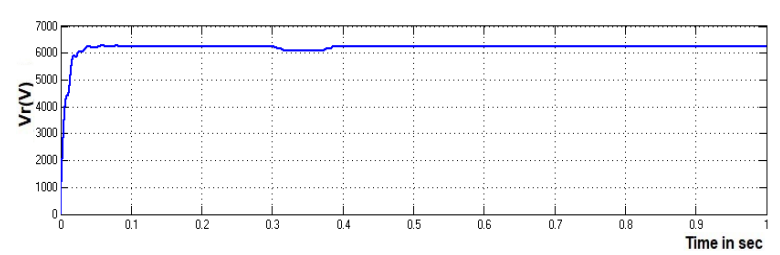

Fig. 9. RMS voltage at bus-31 of the closed loop -UPQC based-T-T-B-S with PR controller

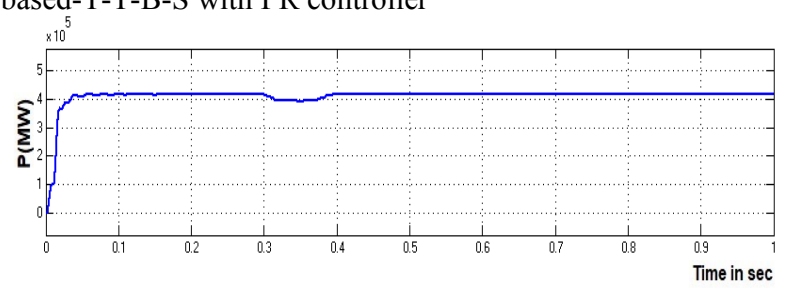

Fig. 10. Real power at bus-31 of the closed loop -UPQC based-T-T-B-S with PR controller

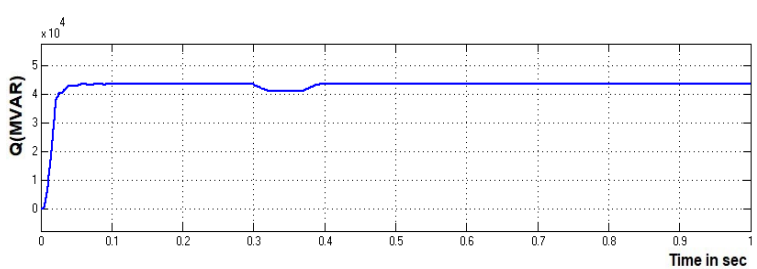

Fig. 11. Reactive power at bus-31 of the closed loop - UPQC based-T-T-B-S with PR controller

\subsection{Closed loop UPQC-TTBS-with MP-controller}

Fig.12 delineates Circuit-Model for C-UPQC-TTBS with MP-controller.Load-value sensed\&it will be evaluated-by-mentioned-voltage to obtain-voltageerrors'. The-voltage-inaccuracy be deviated towardsMPC.Yield of MPC utilized for updating P-W. Fig.13 outlines Voltage at bus-31 of the closed loop-UPQCbased T-T-B-S with MPcontroller. The value of voltage at bus-31 is $0.89 * 10^{4} \mathrm{~V}$. Fig .14 outlines the Current at bus-31 of the closed loop-UPQC-based T-T-B-S with MPcontroller.

The value of Current at bus-31 is 99A. Fig.15 outlines the RMS voltage at bus-31 of the closed loopUPQC-based T-T-B-S with MPcontroller. The value of RMS voltage is $6382 \mathrm{~V}$ and it is stable. Fig. 16 outlines the Real power at bus-31 of the closed loop-UPQCbased T-T-B-S with MPcontroller.Real power-value- is $4.32 * 10^{5} \mathrm{MW} \&$ it is stable. Fig. 17 outlines the Reactive power at bus-31 of the closed loop-UPQC-based T-T-B$\mathrm{S}$ with MPcontroller. The value of Reactive power is $4.38 * 10^{4} \mathrm{MVAR}$ and it is stable.

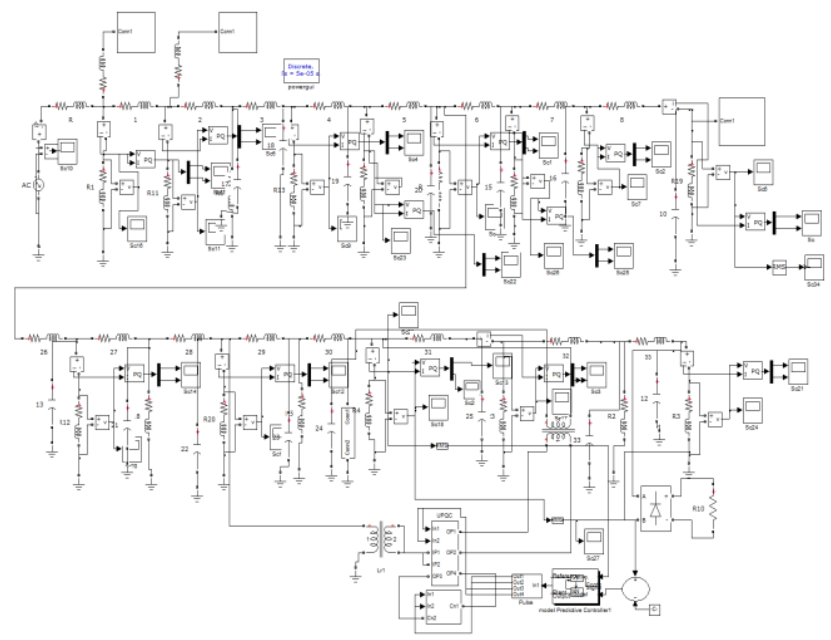

Fig. 12. UPQC based-33BUS with MPC

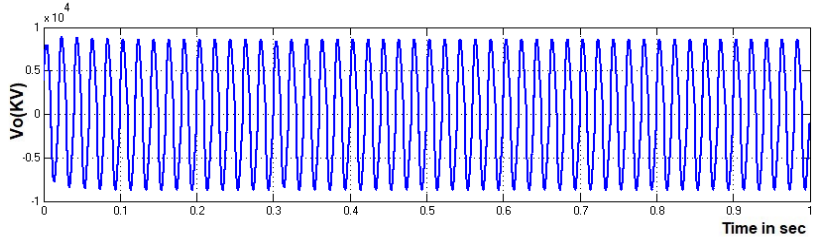

Fig. 13.Voltage at bus-31 for -UPQC based-33B with MPC 


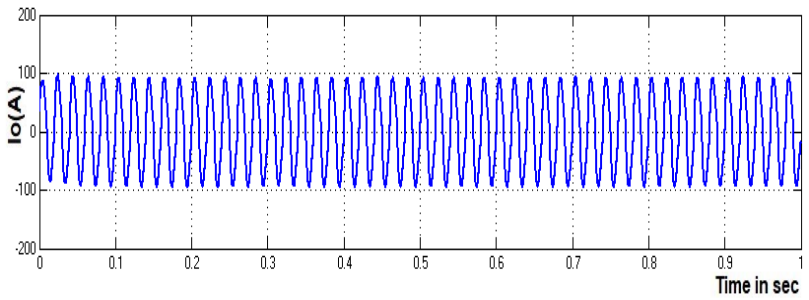

Fig. 14.Current at bus-31 for -UPQC based- MPC

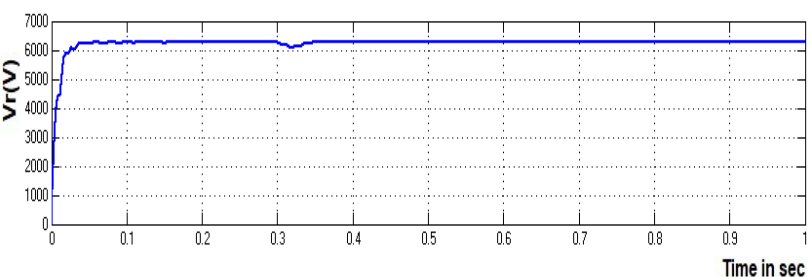

Fig. 15.V-RMS of-UPQC based-TTBS with MPC

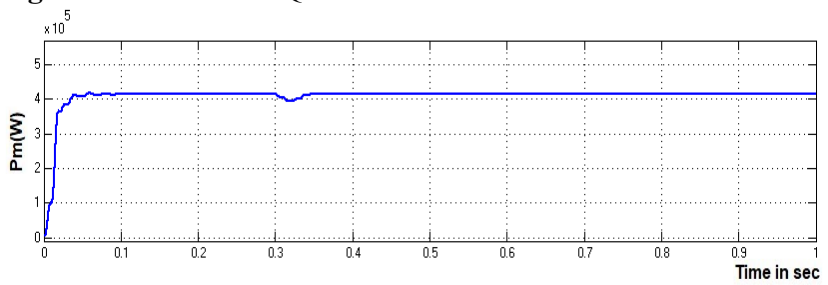

Fig. 16.Real-power at bus 31 for TT-BS with MPC

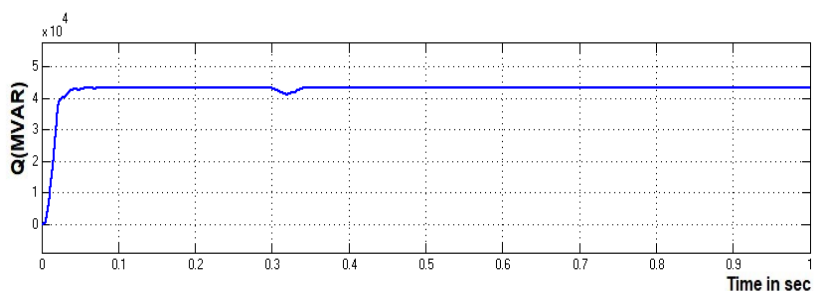

Fig. 17.Reactive-power at bus-31 for TT-BS with MPC

"Comparison-of-time-domain-parameters using PR and MPC" is specified inTable1.By utilizing MPC, -RiseTime is moderated from0.31 Sec to0.306Sec;'Time-Peak' moderates 0.35S-0.32S; Time Settles down' from0.39Sec to $00.34 \mathrm{Sec}$;-Steady-State-Error is diminished from $2.2 \mathrm{~V}$ to $1.1 \mathrm{~V}$. Henceforth, the outcome represents that the closed loop 33- bus U-P-Q-C with MPC is greater thanclosed-loop33- bus U-PQ-C with PRC. Fig.18 delineates the Bar chart presentation with PR-MPC.

Table -1 Comparison Of Time Domain Parameters Using PR-MPC

\begin{tabular}{|c|c|c|c|c|}
\hline Controller & Tr & Tp & Ts & Ess \\
\hline PR & 0.311 & 0.35 & 0.39 & 2.2 \\
\hline MPC & 0.306 & 0.32 & 0.34 & 1.1 \\
\hline
\end{tabular}

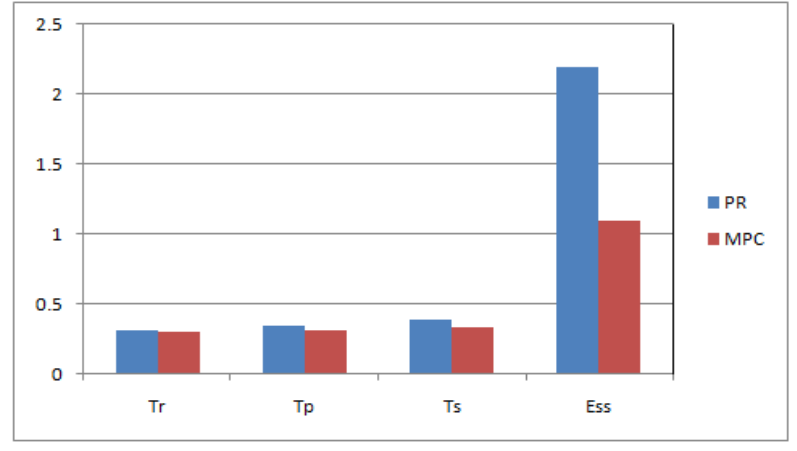

Fig. 18. Bar chart comparison of time domain parameters of the closed loop -UPQC based-T-T-B-S using PR and MPC

\section{Conclusion}

Closed loop U-P-Q-C based 33 bus with PR and MPC schemes will be designed. Outcomes indicates-SettlingTime \&SS-Error to be lowered using MP-controller. By utilizing MPC, -Rise-Time is moderated from0.31Sec to $0.306 \mathrm{Sec}$; time of peak moderated by $00.35 \mathrm{~S}$ to $0.32 \mathrm{~S}$; -Settling-value' goes-from0.39Sec to $0.34 \mathrm{Sec} ;-\mathrm{SSE}$ is decreased from $2.2 \mathrm{~V}-1.1 \mathrm{~V}$. Henceforth, the outcome represents that the closed loop 33- bus U-P-Q-C with MPC is greater than-closed-loop33- bus U-PQ-C with PRC. U-P-Q-C has the capability to reimburse thevoltage-sag-in-power\& dispersal-lines.

\section{References}

1. A.A.A. El-Ela, R.A. El-Sehiemy, A-M Kinawy, et al., "Optimal capacitor placement in distribution systems for power loss reduction and voltage profile improvement," IET Gener. Transm. Distrib., vol. 10, no. 5, pp. 1209-1221, 2016.

2. D. Das, "Optimal placement of capacitors in radial distribution system using Fuzzy-GA method," Int. Journal of Electrical Power and Energy Systems, vol. 30, pp. 361-367, 2008.

3. R. Pegado, Z. Ñaupari, Y. Molina, et al., "Radial distribution network reconfiguration for power losses reduction based on improved selective BPSO," Electric Power Systems Research, vol. 169, pp. 206-213, 2019.

4. S. Ganguly, and D. Samajpati, "Distributed generation allocation on radial distribution networks under uncertainties of load and generation using genetic algorithm," IEEE Transactions on Sustainable Energy, vol. 6, no. 3, pp. 688-697, 2015.

5. A. Ghosh, and G. Ledwitch, "Power quality enhancement using custom power devices," Springer, 2009.

6. H. Fujita, and H. Akagi, "The unified power quality conditioner: The integration of series- and shuntactive filters," IEEE Trans. Power Electronics, vol. 13, no. 2, pp. 315-322, 1998. 
7. V. Khadkikar, "Enhancing electric power quality using UPQC: A Comprehensive review," IEEE Trans. Power Electronics, vol. 27, no. 5, pp. 22842297, 2012.

8. T. Suresh Kumar and V. Sankar, "Reliability improvement of composite Electric power system using Unified Power Flow Controller," 2011 Annual IEEE India Conference, Hyderabad, 2011, pp. 1-6, doi: 10.1109/INDCON.2011.6139523.

9. V. Khadkikar, and A. Chandra, "A control philosophy for a unified power quality conditioner (UPQC) to coordinate load-reactive power demand between series and shunt inverters," IEEE Trans. Power Delivery, vol. 23, no. 4, pp. 2522-2534, 2008.

10. V. Khadkikar, "Fixed and variable power angle control methods for unified power quality conditioner: operation, control and impact assessment on shunt and series inverter $\mathrm{kVA}$ loadings," IET Power Electronics, vol. 6, no. 7, pp. 1299-1307, 2013.

11. R. K. Patjoshi, and K. Mahapatra, "Highperformance unified power quality conditioner using non-linear sliding mode and new switching dynamics control strategy," IET Power Electronics, vol. 10, no. 8, pp. 863-874, 2017.

12. S. Devassy, and B. Singh, "Control of solar photovoltaic integrated UPQC operating in polluted utility conditions," IET Power Electronics, vol. 10, no.12, pp. 1413-1421, 2017.

13. S. Devassy, and B. Singh, "Design and performance analysis of three phase solar PV integrated UPQC," IEEE Trans. Industry Application, vol. 54, no. 1, pp. 73-81, 2018.

14. V. S. Cheung, R. S. Yeung, H. S. Chung, et al., "A transformer-less unified power quality conditioner with fast dynamic control," IEEE Trans. Power Electronics, vol. 33, no. 5, pp. 3926-3937, 2018.

15. J. Ye, H. B. Gooi, and F. Wu, "Optimal design and control implementation of UPQC based on variable phase angle control method," IEEE Trans. Industrial Informatics, vol. 14, no. 7, pp. 3109-3123, 2018.

16. J. Ye, H. B. Gooi, and F. Wu, "Optimization of the size of UPQC system based on data-driven control design," IEEE Trans. Smart Grid, vol. 9, no. 4, pp. 2999-3008, 2018.

17. S. Lakshmi, and S. Ganguly, "Modelling and allocation of open-UPQC integrated PV generation system to improve energy efficiency and power quality of radial distribution networks," IET Renewable Power Generation, vol. 12, no. 5, pp. 605-613, 2018.

18. S. Lakshmi, and S. Ganguly, "Simultaneous optimization of photovoltaic hosting capacity and energy loss of radial distribution networks with open unified power quality conditioner allocation," IET Renewable Power Generation, vol.12, no. 12, pp. 1382-1389, 2018. 